\title{
Volvox and volvocine green algae
}

\author{
James G. Umen * (1)
}

\begin{abstract}
The transition of life from single cells to more complex multicellular forms has occurred at least two dozen times among eukaryotes and is one of the major evolutionary transitions, but the early steps that enabled multicellular life to evolve and thrive remain poorly understood. Volvocine green algae are a taxonomic group that is uniquely suited to investigating the step-wise acquisition of multicellular organization. The multicellular volvocine species Volvox carteri exhibits many hallmarks of complex multicellularity including complete germ-soma division of labor, asymmetric cell divisions, coordinated tissue-level morphogenesis, and dimorphic sexes-none of which have obvious analogs in its closest unicellular relative, the model alga Chlamydomonas reinhardtii. Here, I summarize some of the key questions and areas of study that are being addressed with Volvox carteri and how increasing genomic information and methodologies for volvocine algae are opening up the entire group as an integrated experimental system for exploring the evolution of multicellularity and more.
\end{abstract}

Keywords: Volvox carteri, Chlamydomonas reinhardtii, Multicellularity, Green algae, Morphogenesis, Comparative genomics

\section{Natural habitat and lifecycle}

Volvox is a polyphyletic genus of multicellular freshwater green algae (Chlorophyta) that belong to a larger taxonomic grouping within the Order Chlamydomonadales known as volvocine algae [1]. The species Volvox carteri f. nagariensis (hereafter $V$. carteri or Volvox unless otherwise specified) is the best-studied species in the genus, and has become an established model for developmental and evolutionary biology [2]. Two other $V$. carteri forma that are available in culture collections, f. kawasakiensis and f. weismannia, are closely related but different species from $V$. carteri $\mathrm{f}$. nagariensis. Volvocine genera are cosmopolitan and have been collected on all continents except Antarctica [3, 4]. The habitats of $V$. carteri include small bodies of water (ponds, rice paddies) and larger lakes/reservoirs [3].

While on its own Volvox is a highly tractable model organism, it has even greater utility when it is studied in the context of the volvocine algal clade which includes

*Correspondence: jumen@danforthcenter.org

Donald Danforth Plant Science Center, 975 N. Warson Rd, St. Louis, MO 63132, USA several additional multicellular genera with different sizes, cell numbers and degrees of developmental complexity (e.g., Tetrabaena, Gonium, Astrephomene, Pandorina, Yamagishiella, Eudorina, Pleodorina), and a close unicellular outgroup species, the model alga Chlamydomonas reinhardtii (Chlamydomonas) [2, 5] (Fig. 1). The divergence time between the common ancestor of C. reinhardtii and multicellular volvocine species is estimated at around $250 \mathrm{MY}$ [6], though the fossil record for green algae is sparse and complicated by homoplasy that makes definitive taxonomic affiliation and time calibration difficult [7]. No other group of extant organisms captures the crucial transition of eukaryotic life from unicellular to multicellular as lucidly as the volvocine clade, and much of modern Volvox research is motivated by the potential to reconstruct the early stages of this transition in unprecedented detail $[1,8]$.

Vegetative phase $V$. carteri are spheroids of several hundred microns diameter which are organized in a simple radially symmetric multicellular body plan with just two cell types: 2000 small sterile biflagellate somatic cells that are similar in size to a Chlamydomonas cell, and 12-16 large aflagellate 


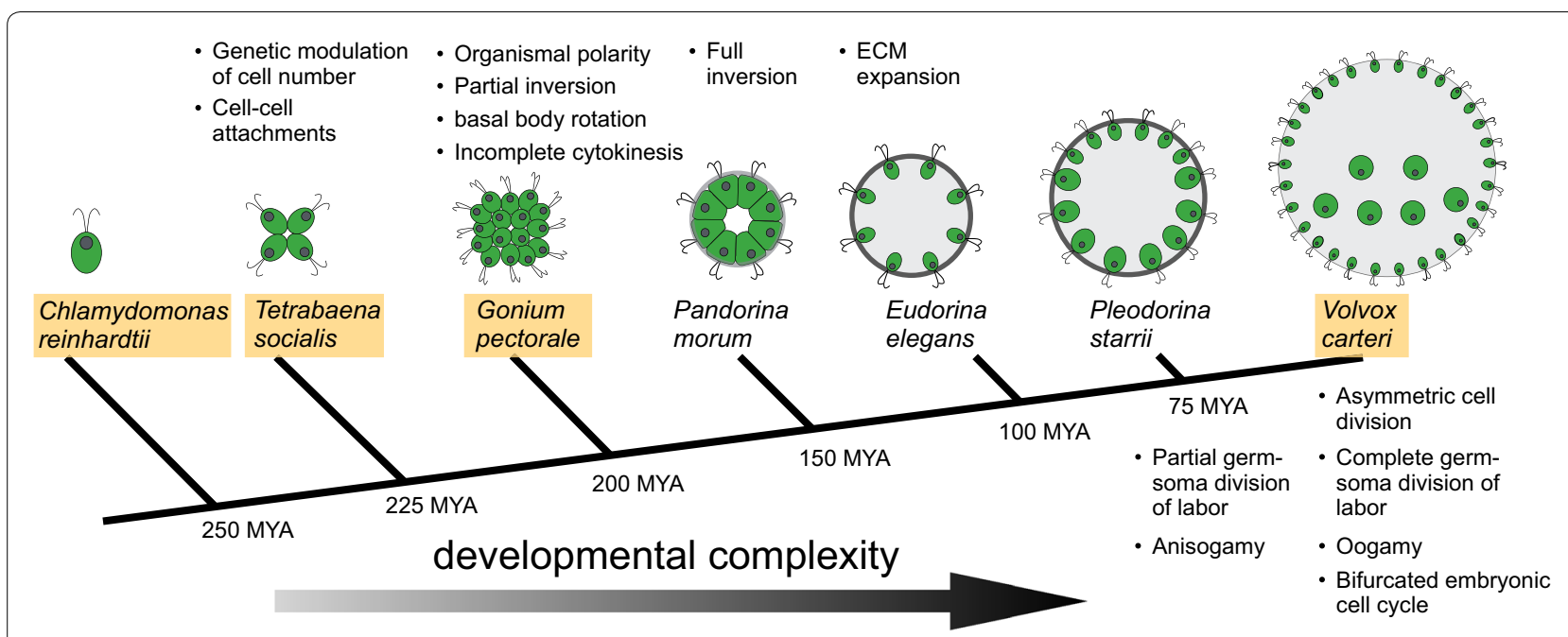

Fig. 1 Volvox and volvocine algae. Simplified cladogram of selected volvocine species from representative genera shown in cartoon form with successive cellular and developmental innovations indicated by bulleted descriptions above or below the node in which they arose. Volvocine algal cells are polarized with an apical pair of flagella just above the nucleus and a single large chloroplast located basally. Species with published sequenced genomes have names in tan boxes. Light grey shading depicts extra cellular matrix (ECM) that occupies increasing amounts of spheroid volume in successive genera. Estimated divergence times at each node are based on data in [6]. This figure was adapted from [92] and reproduced here under Creative Commons Licence CC BY 4.0

reproductive cells called gonidia (Fig. 2a-e). The somatic cells are positioned around the exterior of the spheroid and provide motility, while the gonidia are distributed in the posterior region just below the surface. All of the cells are embedded within a clear secreted glycoprotein-rich extracellular matrix (ECM) that occupies around $99 \%$ of the spheroid volume.

Volvox and all volvocine species have a haplontic life cycle (Fig. 3) where vegetative (i.e., mitotic) haploidphase reproduction can be maintained indefinitely, and where sex is triggered by either nutrient or hormonal signals. Nitrogen deficiency is the cue for sexual differentiation in most volvocine genera, but in Volvox, a species-specific glycoprotein hormone called sex inducer (SI) is the trigger for sex. SI production can be induced by heat shock [9], and when vegetative gonidia are exposed to SI they undergo modified embryogenesis leading to development of sexually dimorphic males or females under the control of a haploid UV sex chromosome system [10-12]. Fertilization produces diploid zygotes which mature into thick-walled dormant zygospores. Zygospores are environmentally resistant and can remain viable for years in a frozen or desiccated state. Exposure of zygospores to light and nutrients initiates germination and meiosis to produce recombinant haploid progeny that re-enter the vegetative life cycle [13]. Detailed descriptions of mating and fertilization can be found elsewhere $[3,14]$.

\section{Lab culture and field collection}

Field samples of Volvox are routinely collected in one of two different ways: Starr and colleagues described a simple means of germinating zygospores from dried soil samples that are usually collected near bodies of water where Volvox has been observed [14]. Dried soil is advantageous as it can be transported and stored easily, though successful germination is never guaranteed. The alternative is collection of live samples using a phytoplankton sieve as demonstrated in this video from Dr. Hisayoshi Nozaki's field studies [15].

A single male-female pair HK9 and HK10 (a.k.a. Adm and Eve) germinated from soil collected near Kobe, Japan, are founders for standard strains still used by most laboratories [3, 14]. The HK9 male strain acquired a morphological mutation and was replaced with a normal male progeny (69-1b) derived from a cross with HK10 [16]. Additional strains, including newer isolates [17, 18], are available from several culture collections (see below). For routine studies female strains (e.g., Eve) are preferred, as males can spontaneously undergo sexual differentiation-an irreversible developmental transition for males whose sperm and somatic cells cannot de-differentiate [3]. Because sexual males produce additional SI, a single spontaneous sexual male arising in a culture can sexually induce the entire population of males, leading to its extinction. Cultures are usually maintained in defined liquid media such as Standard Volvox Medium (SVM) 

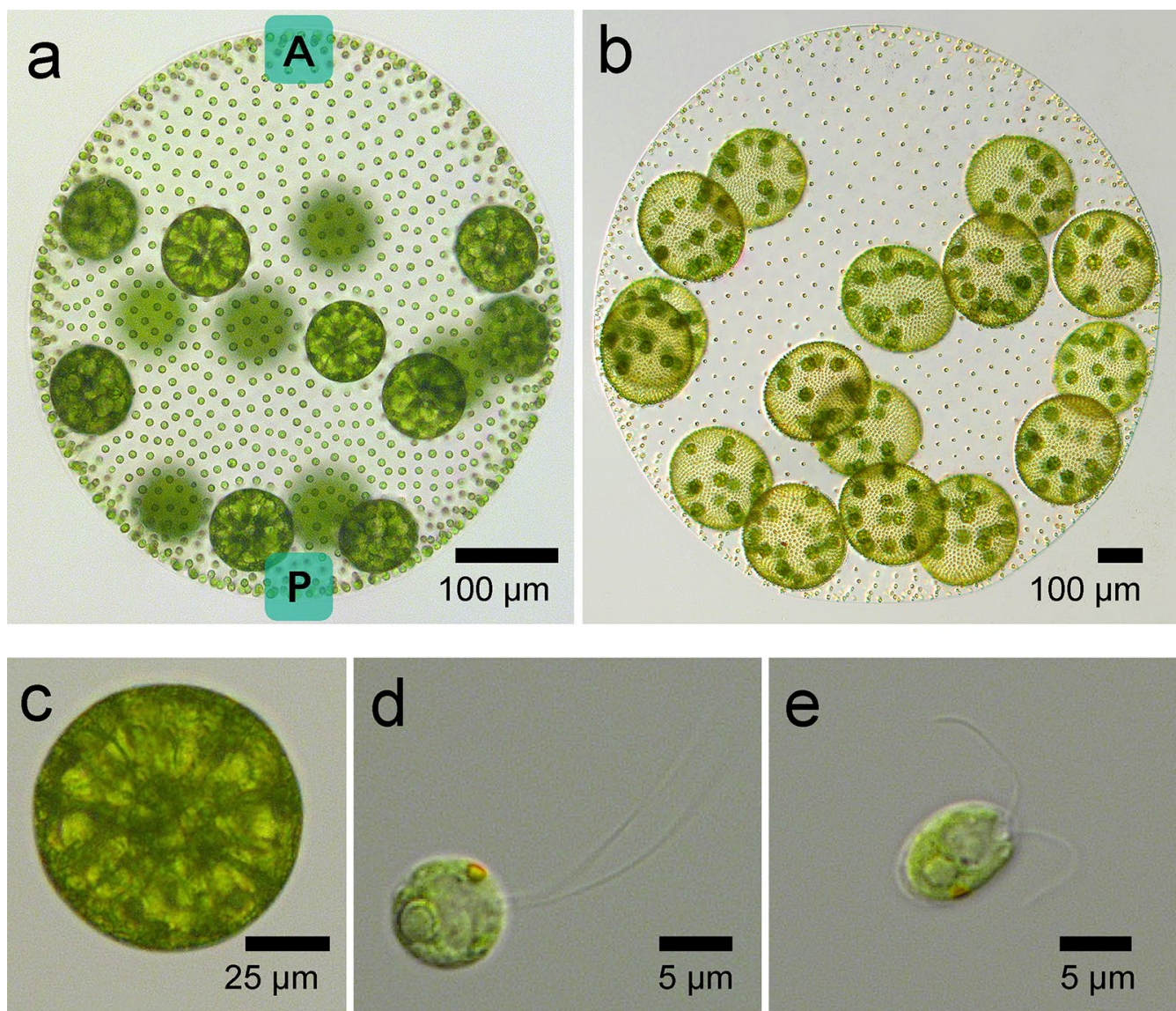

Fig. 2 Volvox carteri. a-d Light micrographs of vegetative phase Volvox carteri (Volvox) showing a mature pre-cleavage stage adult with the anterior (A) and posterior (P) poles labeled (a); a mother spheroid with juveniles (b); an isolated gonidium (c); a somatic cell from a mature pre-cleavage adult spheroid (d). The orange/brown-colored eyespot on the somatic cell is visible near the periphery at around 1 o'clock, and the parallel-oriented flagella are visible near 2 o'clock. e. Light micrograph of a Chlamydomonas reinhardtii cell with flagella oriented to beat in opposite directions. This figure was adapted from [92] and reproduced here under Creative Commons Licence CC BY 4.0

[19] in a temperature- and light-controlled environment, i.e., a plant growth chamber or similar setup (Fig. 4a). Optimal growth is at $32 \mathrm{C}$ under moderately strong light (200-300 $\mu \mathrm{E} \mathrm{m}$-2 s-1 white light, 16 h:8 h L:D cycle), with aeration provided by bubbling (Fig. 4b), and under these or similar conditions the vegetative life cycle can be synchronized, with a new generation produced every $48 \mathrm{~h}$ (Fig. 3). Small-scale stock cultures (20-40 mLs) in glass tubes can be grown without aeration and maintained at room temperature $(\sim 25 \mathrm{C})$ on light shelves or in incubators (Fig. 4a). Maintaining cultures axenically is ideal and requires meticulous sterile technique, but methods exist to remove microbial contaminants. Although there is one report of successful cryopreservation of Volvox [20], maintenance of Volvox cultures generally requires live passaging every 2 to 4 weeks. Dried or frozen zygospores are an alternative method for long-term storage, but this approach cannot maintain a completely isogenic strain background.

\section{Major interest and research questions}

Volvox is a rich developmental system where the origins of developmental complexity and innovation can be inferred using comparative methods and direct experimental testing $[1,5,21,22]$. The late David Kirk, whose laboratory played a major role in ushering Volvox research into the modern molecular era, summarized 12 key steps or innovations in the progression from a unicellular Chlamydomonas-like ancestor to V. carteri [21] (summarized in Fig. 1, with subsequent refinement/elaboration by others $[5,23$, 24]). The earliest innovations include cell adhesion, genetic control of cell number, acquisition of organismal polarity, incomplete cytokinesis with formation of cytoplasmic bridges between embryonic blastomeres, 


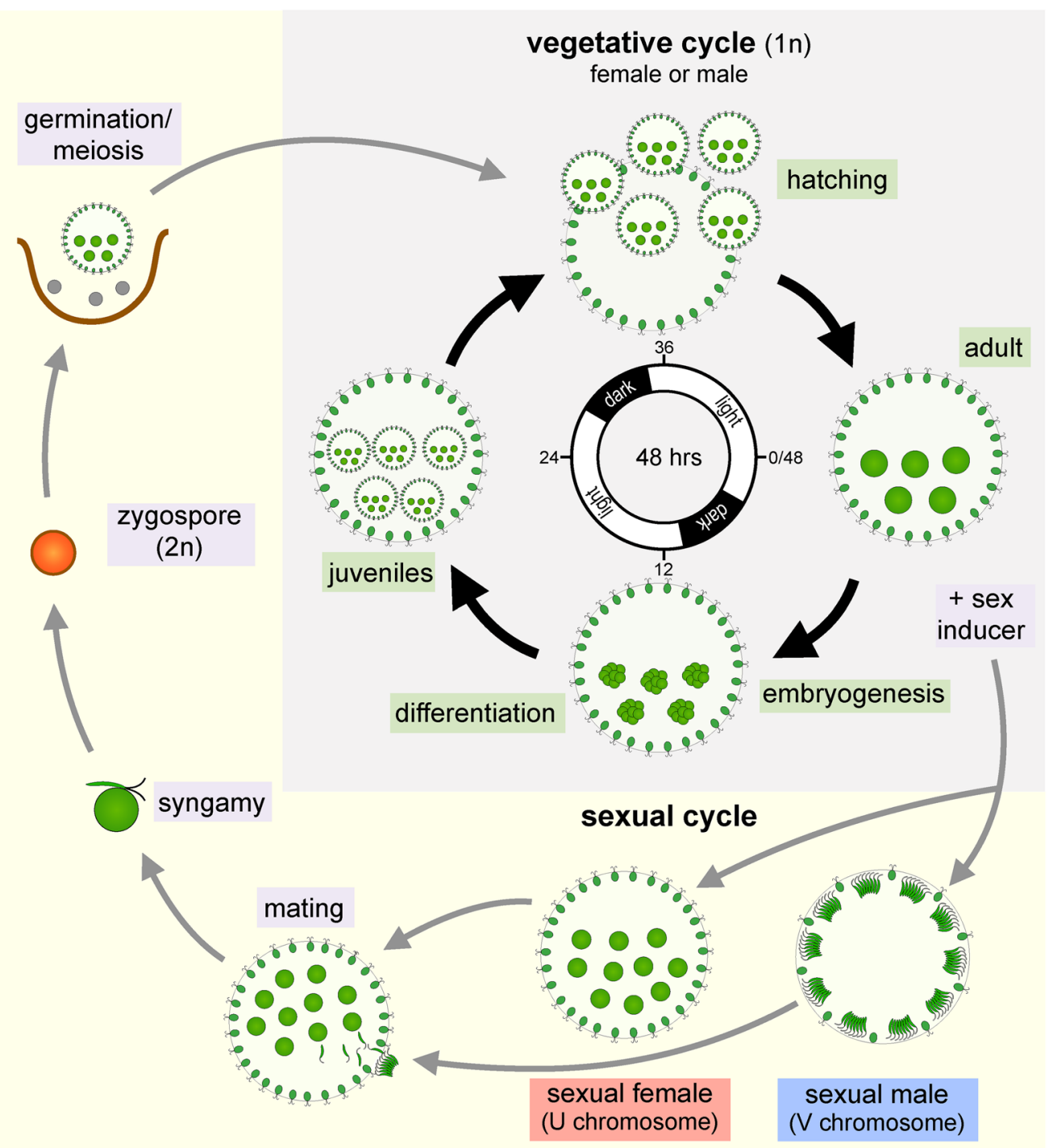

Fig. 3 Volvox life cycle. Schematic of the Volvox vegetative and sexual life cycle phases. Vegetative reproduction (grey shaded region) occurs in the haploid (1n) phase and can be synchronized with a $16 \mathrm{~h}: 8 \mathrm{~h}$ light:dark diurnal regime (center of diagram), under which one full reproductive cycle is completed every $48 \mathrm{~h}$. Vegetative development starts with mature pre-cleavage adults ( 3:00 on diagram, corresponding to spheroid in Fig. 2a) and proceeds clock-wise through embryogenesis and then cyto-differentiation of germ cells (gonidia) and somatic cells to form juveniles ( 9:00 on diagram, corresponding to spheroid in Fig. 2b), hatching of juveniles, and finally maturation to complete the cycle as the next generation of adults. After hatching, the parental somatic cells of the previous generation are discarded and undergo senescence and cell death. Sexual development is triggered by exposure to sex inducer and leads to gonidia undergoing modified embryogenesis and development (not shown) into adult sexual egg-bearing females or adult sexual sperm-packet-bearing males. Sperm packets are released and swim to females where mating takes place with internal fertilization (syngamy), resulting in formation of diploid zygospores. Meiosis occurs upon germination and produces three polar bodies and one haploid progeny that re-enters the vegetative life cycle. This figure was adapted from [10], and reproduced here with permission from the Annual Review of Microbiology, Vol. 73 @ 2019 by Annual Reviews, http://www.annualreviews.org

morphogenetic shape changes (a precursor to the process of inversion described below), rotation of basal bodies to enable coordinated flagellar motility, and conversion of the cell wall to extracellular matrix (ECM) and colony boundary. Subsequent innovations included expansion of the ECM that enabled rapid organismal enlargement, partial and complete germsoma division of labor, asymmetric cell divisions, bifurcated embryonic cell division programs, and anisogamy/oogamy. Deciphering the molecular genetic origins of these steps continues to be an exciting and 


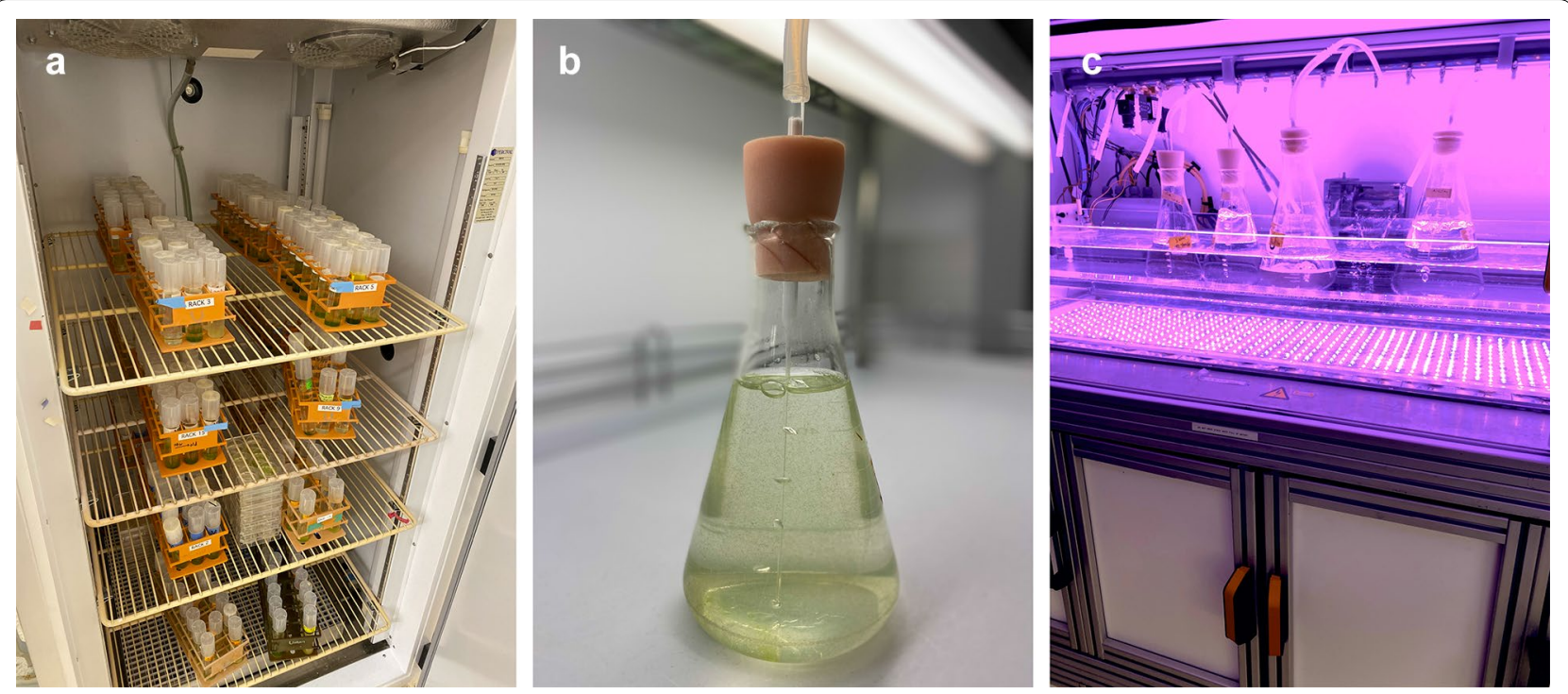

Fig. 4 Volvox cultures. a Stock cultures kept in temperature- and light-controlled growth chamber. b Volvox culture in 500-mL flask plugged with silicone sponge stopper and aerated via cotton-plugged Pasteur pipette. Individual spheroids are just barely visible to the naked eye. $\mathbf{c}$ LED growth chamber for synchronizing cultures. Culture flasks similar to that in panel $\mathbf{b}$ sit in a temperature-controlled acrylic water bath and are lit from below by red and blue LEDs that can be programmed for a diurnal regime. A gas manifold above the flasks provides pressurized air for culture aeration

increasingly approachable goal. A sampling of these innovations and related topics are discussed below in reference to Volvox.

\section{Germ-soma cell-type specialization}

Terminally differentiated somatic cells are a hallmark of complex multicellular organisms and are likely to have arisen independently at least three times in volvocine algae [5]. In $V$. carteri, maintenance of somatic differentiation is governed by a somatic cell-specific SAND domain transcription factor, RegA, which is part of a volvocine algal specific VARL family that underwent a complex history of duplications in multicellular volvocine genera [25-27]. Germ-soma differentiation in V. carteri occurs shortly after embryogenesis is complete, and is dictated by postembryonic cell size; but the coupling between cell size and cell fate remains a mystery [28]. Recent cell-type transcriptome studies in Volvox have also shed light on how germ and somatic cells differ, and the potential origins of their specialized differentiation programs [29-31]. Much remains to be discovered in this system including identifying direct targets of RegA, identification of germ cell-specific differentiation factors, and determining how asymmetric cell divisions governed by the chromatin-associated chaperone proteins glsA, Hsp70A and other factors are specified and executed [32].

\section{Embryogenesis and morphogenesis}

Volvox has evolved some remarkable developmental innovations which have no clear analogs in its unicellular relative, Chlamydomonas. These include asymmetric embryonic cell divisions and a bifurcated embryonic cell division program where both the number of cell divisions and their symmetry differ in a predictable manner among cleaving blastomeres. This developmental bifurcation gives rise to a post-cleavage embryo containing 2000 small somatic-precursor cells arranged like a hollow-ball with 12-16 exterior-facing large gonidialprecursor cells in predictable evenly spaced locations around the embryo's anterior region. The remarkable process of inversion immediately follows embryonic cleavage and involves a sequence of coordinated cell shape changes that enable the embryo to turn itself completely inside-out and assume its adult configuration with outward-oriented somatic precursors and interiorly located gonidial precursors [33]. Anterior-posterior (A-P) embryonic polarity and the presence of cytoplasmic bridges connecting post-mitotic blastomeres are key derived features that enable inversion to occur [34-37]. Mutants that disrupt inversion and other developmental programming have been isolated and hold great potential for understanding the origin of these innovations (reviewed in [2]). 


\section{Evolution of sexes and sex chromosomes}

As described above, Volvox has separate male and female sexes whose differentiation is controlled by a pair of haploid UV sex chromosomes [12] that evolved from an ancestral mating-type locus [10, 11] The entire volvocine lineage has been used as a test case for predictions about how/why anisogamy and oogamy evolved in response to an increase in organismal size and cell number $[10,24]$. The conserved RWP-RK family transcription factor, Mid (minus dominance) resides on the male chromosome (or minus matingtype locus in isogamous species) and controls male or minus sexual differentiation in dioicous species of volvocine algae [10]. Plus mating type or female are the default differentiation programs when Mid is absent. Monoicy (i.e., epigenetic sex determination) is present in some species, but dioicy is likely to be ancestral. In Volvox Mid is inferred to have evolved control of more complex developmental programming than in isogamous volvocine species so that it can promote male sexual differentiation and sperm development [38, 39]. Although Mid controls key aspects of Volvox sexual germ cell differentiation, other genes on the Volvox UV sex chromosomes play additional roles in specifying some aspects of male versus female embryonic patterning and in promoting sex-specific reproductive fitness. This finding and others fit predictions of evolutionary theory [40] where sex chromosomes are postulated to acquire genes required for reproductive fitness of males or females. Other derived aspects of the Volvox sexual cycle such as SI-triggered signal transduction, internal fertilization, and reduced meiotic products are fascinating and relatively unexplored areas of interest [10, 24].

\section{Genome evolution/comparative genomics}

The Chlamydomonas nuclear genome sequence was published in 2007 [41], and the Volvox genome followed in $2010[1,42]$. Two other volvocine nuclear genomes for the multicellular genera, Gonium and Tetrabaena, have also been published [43, 44], with more likely to follow soon. Additional genomic resources including some mating loci sequences, unannotated nuclear genome assemblies, and selected genomic regions are also available but have no public browsers or search engines $[11,25]$. By most metrics volvocine genomes do not differ significantly in size or protein coding capacity, a finding which suggests that developmental innovation occurred mostly through modification or repurposing of a common ancestral genetic "toolkit" [42]. Noncoding genomic regions among the four sequenced species show high divergence, limiting their potential for predicting cis-regulatory elements based on sequence conservation. Additional regulatory complexity derived from microRNAs or small non-coding RNAs may also exist in Chlamydomonas and Volvox, but little is known about how these pathways are involved in basic cellular processes or development [31, $45-47]$.

\section{Motility}

Motility in Volvox and other volvocine species poses interesting developmental, evolutionary and biophysical questions [48-50]. Volvox swims while rotating about its A-P axis which is oriented in the direction of swimming (Fig. 2). To achieve coordinated swimming and phototaxis, somatic cells must be oriented correctly along the A-P axis, with their two flagella beating in parallel-a configuration that is achieved through each basal body rotating $90^{\circ}$ about its long axis from a starting $180^{\circ}$ orientation present in newly formed somatic cells. The $180^{\circ}$ basal body orientation is an ancestral configuration that was retained in unicellular relatives such as Chlamydomonas which swims using a breast-stroke motion (Fig. 2d and 2e)[51, 52]. Flagellar responses to light in each somatic cell are mediated by an eyespot-an organelle containing channel rhodopsin photoreceptors which are shielded from light on one side by carotenoidrich pigment granules that visibly mark the eyespot as a brown/orange dot on the side of the cell (Fig. 2d). This configuration enables the channel rhodopsin in each eyespot to act as directional light sensor whose open/closed state transitions are tuned to allow synchronization between swimming rotation frequency and flagella beating responses mediated by calcium signaling. Together, the eyespots act as a distributed sensing system allowing the flagella of somatic cells on the side of the spheroid rotating past the path of incoming light to transiently adjust their beat-stroke and effect a turn towards or away from the light [53-55].

\section{Experimental approaches}

Volvox is amenable to many types of experimental manipulations and methodologies. Because it can be easily synchronized using a diurnal regime, uniform populations from different developmental or life cycle stages can be obtained. At many (but not all) stages the germ and somatic cells (or cleaving embryos) can be physically separated and purified, thus enabling studies of individual cell types $[29,30]$. Pre-cleavage or cleaving embryos can be released from parental spheroids. They remain viable and can complete development, allowing them to be studied in detail using time lapse imaging, or can be subject to various experimental manipulations [56]. Volvox is amenable to most standard methods of microscopic 
imaging including single plane illumination microscopy (SPIM, a.k.a. light sheet microscopy) to study development and morphogenesis [33, 57], and video microscopy to study motility and flagella dynamics. A recent study was able to establish in vitro motility in "zombie" Volvox whose cells were permeabilized but whose flagella could be re-activated in the presence of ATP and calcium to investigate flagella responses to calcium signaling [54].

Molecular genetics methods for Volvox make it a highly tractable system for developmental genetics. Classical genetics played a critical role in defining different classes of developmental mutants [58-62], but was superseded by the more facile method of transposon tagging which was the method of choice prior to having a genome sequence [35, 63-65]. With a sequenced genome, isolates that have abundant sequence polymorphisms [66], and low-cost resequencing methods, the prospects for using classical developmental genetics are again excellent. It should be noted that strains tend to lose sexuality during repeated vegetative propagation, but this can be remedied by routine crossing to maintain sexual fitness.

Volvox can be transformed using biolistic methods (DNA-coated gold particles delivered with a helium gene gun) coupled with any of several available selection markers [67-71]. Nuclear transgenes are thought to integrate randomly as they do in Chlamydomonas, and can be subject to silencing and position effects (i.e., influence on expression by nearby endogenous loci) [72]. During selection the spheroids grow for one or more generations, with non-transformed individuals eventually dying, and stably transformed genetically homogenous transformants emerging. Although overall transformation frequency is not high (typically several to a few dozen per biolistic shot targeting $\sim 30,000$ cleaving embryos, each with up to 16 germline precursor cells), co-transformation efficiency is quite high (typically $20-80 \%$ ) making it relatively straight forward to express a gene of interest when it is co-bombarded with a selectable marker gene [70]. Regulated promoters have also been described [73, 74]. RNAi-mediated knockdowns using antisense [75, 76] or hairpin constructs [39] have been successful for reverse genetics and will likely remain useful for targeting essential genes where a knockout might be lethal, but will likely be replaced by CRISPR-Cas9 (or similar) genome editing that was successfully adapted for Volvox [77].

Additional methods or advances that would positively impact Volvox research include a reliable method for cryopreservation, more efficient transformation methods, a means of inserting transgenes at defined locations, and non-endogenous regulated promoters/transcription factors that can be controlled with small molecules or other non-physiological stimuli. Adapting CRISPRCas9-based genome editing to make targeted mutations was a huge breakthrough [77], and the added ability to do more directed and defined genome editing (e.g., creating site specific mutations, adding epitope tags, or creating fusion proteins transcribed from endogenous loci) would open up additional avenues of experimentation.

While Volvox carteri and Chlamydomonas reinhardtii have well-established molecular genetic methods, most other volvocine algae have few or no tools, with successful transformation reported for a handful of other species [78-81]. With more interest and improved genomics resources catalyzed by inexpensive genome and transcriptome sequencing, these current limitations can be overcome and will help further elevate the entire clade as a premier model for comparative and mechanistic studies of evolution and development.

\section{Research community and resources}

Around a dozen laboratories worldwide use Volvox or its multicellular relatives, while the Chlamydomonas community is considerably larger. A biennial International Volvox Conference is held in odd years, with the most recent one in Tokyo, Japan in 2019 [82]. The Volvox meeting is staggered with a biennial International Chlamydomonas meeting held in even years in which Volvox and volvocine algae research are included [83]. Phytozome $[84,85]$ hosts data for the V. carteri and C. reinhardtii genomes and has some useful browser capabilities for viewing external data. Phycosm [86] hosts the former two species plus Gonium pectorale, while the Tetrabaena socialis genome assembly is not currently hosted in the public domain [44]. As mentioned above, there are some additional genomes or parts of genomes available, but with no browsers and/or limited annotations available $[11,25]$. Key culture collections for obtaining Volvox and volvocine algal strains and/or media recipes are UTEX in the US [87], NIES in Japan [88] and SAG in Germany [89]. Excellent video microscopy of Volvox and its relatives can be found online [90] from Jeremy Pickett-Heaps and colleagues. A short list of recommended reading for learning more about different aspects of Volvox biology includes these references: [2, 3, 8, 10, 48, 49, 91].

Abbreviations

Hr: Hour; ECM: Extracellular matrix; SI: Sex inducer; A-P: Anterior-posterior.

Acknowledgements

The micrographs used in Fig. 2 were produced by Gavriel Matt.

Authors' contributions

This review was written by JGU. The author read and approved the final manuscript. 


\section{Funding}

Research support to JGU for Volvox comes from National Science Foundation Grant IOS 1755430.

\section{Availability of data and materials}

Not applicable.

\section{Ethics approval and consent to participate}

Not applicable.

\section{Consent for publication}

Not applicable.

\section{Competing interests}

None.

Received: 30 January 2020 Accepted: 11 June 2020

Published online: 01 July 2020

\section{References}

1. Umen JG, Olson BJSC. Genomics of Volvocine Algae. Piganeau G, editor. Adv Bot Res. 2012;64:185-243.

2. Matt G, Umen JG. Volvox: a simple algal model for embryogenesis, morphogenesis and cellular differentiation. Develop Biol. 2016;419:99-113.

3. Kirk DL. Volvox. Bard JBL, Barlow PW, Green P B, Kirk DL, editors. Cambridge University Press; 1998.

4. Smith GM. A Comparative Study of the Species of Volvox. T Am Microsc Soc. 1944;63:265.

5. Herron MD, Michod RE. Evolution of complexity in the volvocine algae: transitions in individuality through Darwin's eye. Evolution. 2008:62:436-51.

6. Herron MD, Hackett JD, Aylward FO, Michod RE. Triassic origin and early radiation of multicellular volvocine algae. Proc Natl Acad Sci USA. 2009;106:3254-8.

7. Butterfield NJ, Knoll AH, Swett K. Paleobiology of the Neoproterozoic Svanbergfjellet Formation. Spitsbergen Lethaia. 1994;27:76-76.

8. Herron MD. Origins of multicellular complexity: volvox and the volvocine algae. Mol Ecol. 2016:25:1213-23.

9. Kirk D, Kirk M. Heat shock elicits production of sexual inducer in Volvox. Science. 1986:231:51-4

10. Umen J, Coelho S. Algal sex determination and the evolution of anisogamy. Annu Rev Microbiol. 2019:73:1-25.

11. Hamaji T, Kawai-Toyooka H, Uchimura H, Suzuki M, Noguchi H, Minakuchi $Y$, et al. Anisogamy evolved with a reduced sex-determining region in volvocine green algae. Commun Biol. 2018;1:17.

12. Ferris P, Olson BJSC, Hoff PLD, Douglass S, Casero D, Prochnik S, et al. Evolution of an expanded sex-determining locus in Volvox. Science. 2010;328:351-4.

13. Starr RC. Meiosis in Volvox carteri f. nagariensis. Archiv fur Protistenkunde. 1975;117:187-91

14. Starr RC. Structure, reproduction and differentiation in Volvox carterif nagariensis lyengar, strains HK9 \& 10. Arch Protistenkd. 1969;111:204-22.

15. Seining for algae in Lake Biwa [Internet]. n.d. https://youtu.be/aC90_ GuYq4.

16. Meredith RF, Starr RC. The Genetic Basis of Male Potency in Volvox carterif nagariensis (Chlorophyceae). J Phycol. 1975;11:265-72.

17. Nozaki H, Ueki N, Takusagawa M, Yamashita S, Misumi O, Matsuzaki R, et al. Morphology, taxonomy and mating-type loci in natural populations of Volvox carteri in Taiwan. Botanical Studies. 2018;59:10.

18. Kawafune K, Hongoh Y, Hamaji T, Sakamoto T, Kurata T, Hirooka S, et al. Two different rickettsial bacteria invading Volvox carteri. PLOS ONE. 2015:10:e0116192.

19. Kirk DL, Kirk MM. Protein synthetic patterns during the asexual life cycle of Volvox carteri. Dev Biol [Internet]. 1983. 96:493-506. http://eutils.ncbi. nlm.nih.gov/entrez/eutils/elink.fcgi?dbfrom=pubmed\&id=68324 80\&retmode $=$ ref\& $\mathrm{cmd}=$ prlinks

20. Nakazawa A, Nishii I. Amidic and acetonic cryoprotectants improve cryopreservation of volvocine green algae. Cryo Lett. 2012;33:201-12.
21. Kirk DL. A twelve-step program for evolving multicellularity and a division of labor. BioEssays. 2005;27:299-310.

22. Umen JG. Green Algae and the Origins of Multicellularity in the Plant Kingdom. Cold Spring Harbor Perspectives Biol. 2014;6:1-27.

23. Arakaki Y, Kawai-Toyooka H, Hamamura Y, Higashiyama T, Noga A, Hirono $\mathrm{M}$, et al. The simplest integrated multicellular organism unveiled. PloS ONE. 2013:8:e81641.

24. Hanschen ER, Herron MD, Wiens JJ, Nozaki H, Michod RE. Multicellularity drives the evolution of sexual traits. Am Nat. 2018;192:E93-105.

25. Grochau-Wright ZI, Hanschen ER, Ferris PJ, Hamaji T, Nozaki H, Olson BJSC, et al. Genetic basis for soma is present in undifferentiated volvocine green algae. J Evolu Biol. 2017;30:1205-18.

26. Duncan L, Nishii I, Howard A, Kirk D, Miller SM. Orthologs and paralogs of regA, a master cell-type regulatory gene in Volvox carteri. Curr Genet. 2006;50:61-72.

27. Duncan L, Nishii I, Harryman A, Buckley S, Howard A, Friedman NR, et al. The VARL Gene family and the evolutionary origins of the master celltype regulatory gene, regA, in Volvox carteri. J Mol Evol. 2007;65:1-11.

28. Kirk MM, Ransick A, McRae SE, Kirk DL. The relationship between cell size and cell fate in Volvox carteri. The Journal of Cell Biology [Internet]. 1993. 123:191-208. http://eutils.ncbi.n/m.nih.gov/entrez/eutils/elink.fcgi?dbfro $m=$ pubmed\&id $=8408198 \&$ retmode $=$ ref\& $\mathrm{cmd}=$ prlinks.

29. Matt GY, Umen JG. Cell-type transcriptomes of the multicellular green alga Volvox carteri yield insights into the evolutionary origins of germ and somatic differentiation programs. G3. 2018:8:531-50.

30. Klein B, Wibberg D, Hallmann A. Whole transcriptome RNA-Seq analysis reveals extensive cell type-specific compartmentalization in Volvox carteri. BMC Biol. 2017;15:111.

31. Dueck A, Evers M, Henz SR, Unger K, Eichner N, Merkl R, et al. Gene silencing pathways found in the green alga Volvox carteri reveal insights into evolution and origins of small RNA systems in plants. BMC Genomics. 2016. https://doi.org/10.1186/s12864-016-3202-4.

32. Cheng Q, Pappas V, Hallmann A, Miller SM. Hsp70A and GlsA interact as partner chaperones to regulate asymmetric division in Volvox. Develop Biol. 2005:286:537-48.

33. Haas PA, Goldstein RE. Embryonic inversion in Volvox carteri: the flipping and peeling of elastic lips. Phys Rev E. 2018;98:052415.

34. Hoops HJ, Nishii I, Kirk DL. Cytoplasmic Bridges in Volvox and Its Relatives. 2006. p. 65-84

35. Kirk DL, Nishii I. Volvox carteri as a model for studying the genetic and cytological control of morphogenesis. Dev Growth Differ. 2001;43:621-31.

36. Green KJ, Viamontes GI, Kirk DL. Mechanism of formation, ultrastructure, and function of the cytoplasmic bridge system during morphogenesis in Volvox. J Cell Biology [Internet]. 1981. 91:756-69. http://jcb.rupress.org/ content/91/3/756.long.

37. Viamontes $\mathrm{Gl}$, Kirk DL. Cell shape changes and the mechanism of inversion in Volvox. Journal Cell Biol. 1977:75:719-30.

38. Geng S, Miyagi A, Umen JG. Evolutionary divergence of the sex-determining gene MID uncoupled from the transition to anisogamy in volvocine algae. Development. 2018. https://doi.org/10.1242/dev.162537.

39. Geng S, Hoff PD, Umen JG. Evolution of sexes from an ancestral matingtype specification pathway. PLoS Biol. 2014;12:e1001904.

40. Coelho SM, Gueno J, Lipinska AP, Cock JM, Umen JG. UV chromosomes and haploid sexual systems. Trends Plant Sci. 2018;23:794-807.

41. Merchant SS, Prochnik SE, Vallon O, Harris EH, Karpowicz SJ, Witman GB, et al. The Chlamydomonas genome reveals the evolution of key animal and plant functions. Science [Internet]. 2007. 318:245-50. http://www. sciencemag.org/cgi/content/full/318/5848/245/DC1.

42. Prochnik SE, Umen J, Nedelcu AM, Hallmann A, Miller SM, Nishii I, et al. Genomic analysis of organismal complexity in the multicellular green alga Volvox carteri. Science. 2010;329:223-6.

43. Hanschen ER, Marriage TN, Ferris PJ, Hamaji T, Toyoda A, Fujiyama A, et al. The Gonium pectorale genome demonstrates co-option of cell cycle regulation during the evolution of multicellularity. Nat Commun. 2016;7:11370.

44. Featherston J, Arakaki Y, Hanschen ER, Ferris PJ, Michod RE, Olson BJSC, et al. The 4-celled Tetrabaena socialis nuclear genome reveals the essential components for genetic control of cell number at the origin of multicellularity in the volvocine lineage. Mol Biol Evol. 2017;35(4):855-70.

45. Li J, Wu Y, Qi Y. MicroRNAs in a multicellular green alga Volvox carteri. Sci China Life Sci. 2014:57:36-45. 
46. Zhao T, Li G, Mi S, Li S, Hannon GJ, Wang X-J, et al. A complex system of small RNAs in the unicellular green alga Chlamydomonas reinhardtii. Genes Develop. 2007;21(10):1190-203.

47. Molnár A, Schwach F, Studholme DJ, Thuenemann EC, Baulcombe DC. miRNAs control gene expression in the single-cell alga Chlamydomonas reinhardtii. Nature. 2007;447(7148):1126-9.

48. Ueki N, Matsunaga S, Inouye I, Hallmann A. How 5000 independent rowers coordinate their strokes in order to row into the sunlight: phototaxis in the multicellular green alga Volvox. BMC Biology [Internet]. 2010;8:121. http://www.biomedcentral.com/1741-7007/8/103.

49. Goldstein RE. Green algae as model organisms for biological fluid dynamics. Annual Rev Fluid Mech. 2015;47:343-75.

50. Hoops HJ. Motility in the colonial and multicellular Volvocales: structure, function, and evolution. Protoplasma. 1997:199:99-112.

51. Hoops H. Flagellar, cellular and organismal polarity in Volvox carteri. Journal of Cell Science [Internet]. 1993. 104:105-17. http://jcs.biologists.org/ cgi/content/abstract/104/1/105.

52. Hoops HJ. Somatic cell flagellar apparatuses in two species of Volvox (Chlorophyceae). J Phycol. 1984;20:20-7.

53. Drescher K, Goldstein RE, Tuval I. Fidelity of adaptive phototaxis. Proc National Acad Sci. 2010;107:11171-6.

54. Ueki N, Wakabayashi K-I. Detergent-extracted Volvox model exhibits an anterior-posterior gradient in flagellar Ca2 + sensitivity. Proc Natl Acad Sci U S A. 2018:115:E1061-8.

55. Hegemann P. Vision in microalgae. Planta. 1997;203:265-74

56. Ueki N, Nishii I. Controlled enlargement of the glycoprotein vesicle surrounding a volvox embryo requires the InvB nucleotide-sugar transporter and is required for normal morphogenesis. The Plant Cell [Internet]. 2009;21:1166-81. http://www.plantcell.org/cgi/content/full/21/4/1166.

57. Höhn S, Honerkamp-Smith AR, Haas PA, Trong PK, Goldstein RE. Dynamics of a Volvox embryo turning itself inside out. Physical Rev Lett. 2015;114:178101-5.

58. Sessoms AH, Huskey RJ. Genetic control of development in Volvox: isolation and characterization of morphogenetic mutants. Proc Natl Acad Sci U S A. 1973;70:1335-8.

59. Huskey RJ. Mutants affecting vegetative cell orientation in Volvox carteri. Develop Biol. 1979;72:236-43.

60. Huskey RJ, Griffin BE, Cecil PO, Callahan AM. A Preliminary Genetic Investigation of Volvox carteri. Genetics [Internet]. 1979. 91:229-44. http://www. genetics.org/cgi/reprint/91/2/229.

61. Pall ML. Mutants of Volvox showing premature cessation of division: evidence for a relationship between cell size and reproductive cell differentiation. In: McMahon D, Fox CF, editors. 1975. p. 148-56.

62. Callahan AM, Huskey RJ. Genetic control of sexual development in Volvox. Develo Biol. 1980;80:419-35.

63. Kirk MM, Stark K, Miller SM, Müller W, Taillon BE, Gruber H, et al. regA, a Volvox gene that plays a central role in germ-soma differentiation, encodes a novel regulatory protein. Development [Internet] 1999:126:639-47. http://eutils.ncbi.nlm.nih.gov/entrez/eutils/elink .fcgi?dbfrom = pubmed\&id $=9895312 \&$ retmode $=$ ref\&cmd $=$ prlinks.

64. Miller SM, Kirk DL. glsA, a Volvox gene required for asymmetric division and germ cell specification, encodes a chaperone-like protein. Development 1999:126:649-58

65. Ueki N, Nishii I. Idaten is a new cold-inducible transposon of Volvox carteri that can be used for tagging developmentally important genes. Genetics [Internet]. 2008. 180:1343-53. http://eutils.ncbi.nlm.nih.gov/entrez/eutils/ elink.fcgi?dbfrom $=$ pubmed\&id $=18791222 \&$ retmode $=$ ref\&cmd $=$ prlinks.

66. Adams CR, Stamer KA, Miller JK, McNally JG, Kirk MM, Kirk DL. Patterns of organellar and nuclear inheritance among progeny of two geographically isolated strains of Volvox carteri. Current Genetics [Internet]. 1990. 18:141-53. http://eutils.ncbi.nlm.nih.gov/entrez/eutils/elink.fcgi?dbfro $\mathrm{m}=$ pubmed\&id $=1977526 \&$ retmode $=$ ref\& $\mathrm{cmd}=$ prlinks

67. Ortega-Escalante JA, Kwok O, Miller SM. New Selectable Markers for Volvox carteri transformation. Protist. 2018:170:52-63.

68. Jakobiak T, Mages W, Scharf B, Babinger P, Stark K, Schmitt R. The bacterial paromomycin resistance gene, aphH, as a dominant selectable marker in Volvox carteri. Protist. 2004;155:381-93.
69. Gruber H, Kirzinger SH, Schmitt R. Expression of the Volvox gene encoding nitrate reductase: Mutation-dependent activation of cryptic splice sites and intron-enhanced gene expression from a cDNA. Plant Mol Biol [Internet]. 2007. 31:1-12. http://www.springerlink.com/index/U442X 2215K077851.pdf

70. Schiedlmeier B, Schmitt R, Muller W, Kirk MM, Gruber H, Mages W, et al. Nuclear transformation of Volvox carteri. Proc National Acad Sci [Internet]. 1994. 91:5080-4. http://eutils.ncbi.nlm.nih.gov/entrez/eutils/elink fcgi?dbfrom $=$ pubmed\&id $=8197189 \&$ retmode $=$ ref\&cmd $=$ prlinks.

71. Hallmann A, Rappel A. Genetic engineering of the multicellular green alga Volvox: a modified and multiplied bacterial antibiotic resistance gene as a dominant selectable marker. Plant J. 1999;17:99-109.

72. Babinger P, Kobl I, Mages W, Schmitt R. A link between DNA methylation and epigenetic silencing in transgenic Volvox carteri. Nucleic Acids Research [Internet]. 2001;29:1261-71. http://nar.oxfordjournals.org/cgi/ content/abstract/29/6/1261.

73. Hallmann A, Sumper M. Reporter genes and highly regulated promoters as tools for transformation experiments in Volvox carteri. Proc National Acad Sci. 1994;91:11562-6.

74. von der Heyde EL. The inducible nitA promoter provides a powerful molecular switch for transgene expression in Volvox carteri. BMC Biotechnol. 2015;15:1-13.

75. Cheng Q, Hallmann A, Edwards L, Miller SM. Characterization of a heat-shock-inducible hsp70 gene of the green alga Volvox carteri. Gene. 2006;371:112-20.

76. Ebnet E, Fischer M, Deininger W, Hegemann P. Volvoxrhodopsin, a lightregulated sensory photoreceptor of the spheroidal green Alga Volvox carteri. Plant Cell. 1999;11:1473-84.

77. Ortega-Escalante JA, Jasper R, Miller SM. CRISPR/Cas9 mutagenesis in Volvox carteri. Plant J. 2019;97:661-72.

78. Lerche K, Hallmann A. Stable nuclear transformation of Eudorina elegans. BMC biotechnology [Internet]. 2013. 13:11. http://www.biomedcentral. com/1472-6750/13/11/abstract.

79. Lerche K, Hallmann A. Stable nuclear transformation of Gonium pectorale. BMC Biotechnol. 2009;9:64.

80. Lerche K, Hallmann A. Stable nuclear transformation of Pandorina morum. BMC biotechnology. 2014;14:65

81. Grochau-Wright Z. The Origin and Evolution of the REG Cluster in the Volvocine Green Algae: A Model System for the Evolution of Cellular Differentiation. 2019.

82. th International Volvox Conference [Internet]. www.bs.s.u-tokyo.ac. jp/ tayousei/Nolvox2019/index.html.

83. 19th International Conference on the Cell and Molecular Biology of Chlamydomonas [Internet]. n.d. https://chlamy2020.sciencesconf.org/.

84. Goodstein DM, Shu S, Howson R, Neupane R, Hayes RD, Fazo J, et al. Phytozome: a comparative platform for green plant genomics. Nucleic Acids Res. 2012:40:D1178-86.

85. Phytozome [Internet]. https://phytozome.jgi.doe.gov.

86. PhycoCosm [Internet]. https://phycocosm.jgi.doe.gov.

87. UTEX Culture Collection of Algae [Internet]. https://utex.org.

88. Microbial Culture Collection at the National Institute for Environmental Studies [Internet]. https://mcc.nies.go.jp.

89. The Culture Collection of Algae at Goettingen University [Internet]. https ://www.uni-goettingen.de/en/45175.html.

90. Picket-Heaps J. The Volvocales and Chlorococcales [Internet]. https:// www.kanopy.com/product/remarkable-plants-volvocales-and-chlorococ.

91. Miller SM. Volvox, Chlamydomonas, and the evolution of multicellularity. Nat Edu. 2010;3:65.

92. Russell JJ, Theriot JA, Sood P, Marshall WF, Landweber LF, Fritz-Laylin L, et al. Non-model model organisms. BMC Biol. 2017;15:55.

\section{Publisher's Note}

Springer Nature remains neutral with regard to jurisdictional claims in published maps and institutional affiliations. 